Egyptian Journal of Aquatic Biology \& Fisheries

Zoology Department, Faculty of Science,

Ain Shams University, Cairo, Egypt.

ISSN $1110-6131$

Vol. 24(6): 361 - 382 (2020)

www.ejabf.journals.ekb.eg

\title{
Epidermal mucus as a potential biological matrix for fish health analysis
}

\section{Heri B. Santoso ${ }^{1,2 *}$, Eko Suhartono ${ }^{3}$, Rizmi Yunita ${ }^{4}$, and Danang Biyatmoko ${ }^{5}$}

${ }^{1 .}$ Doctoral Department of Agricultural Science, Lambung Mangkurat University. Banjarbaru, South Kalimantan 70714, Indonesia

${ }^{2 .}$ Department of Biology, Faculty of Mathematics and Natural Sciences, Lambung Mangkurat University. Banjarbaru, South Kalimantan, Indonesia

3. Department of Medical Chemistry/Biochemistry, Faculty of Medicine, Lambung Mangkurat University. Banjarbaru, South Kalimantan, Indonesia

4. Department of Aquatic Resources Management, Faculty of Fisheries and Marine Sciences, Lambung Mangkurat University. Banjarbaru, South Kalimantan, Indonesia

5. Department of Animal Science, Faculty of Agriculture, Lambung Mangkurat University. Banjarbaru, South Kalimantan, Indonesia

* Corresponding Author: heribudisantoso@ulm.ac.id

\section{ARTICLE INFO}

Article History:

Received: July 29, 2020

Accepted: Sept. 11, 2020

Online: Sept. 13, 2020

\section{Keywords:}

Fish skin mucus,

Mucus bioactivities,

Mucosal barrier,

Mucosal immunity,

Mucosal biomarker,

Non-invasive biomarker,

Stress

\section{ABSTRACT}

Epidermal mucus serves as a physiological and immunological first line of defense to maintain normal physiological status in Teleost fish. It offers protection against pathogenic infections and environmental contaminants. The ability of mucus as a protector depends on its bioactive components, such as high-molecular-weight glycoproteins, lysozyme, alkaline phosphatase, immunoglobulin, complement proteins, C-reactive proteins, lectins, agglutinin, interferon, vitellogenin, proteases, antimicrobial proteins, antimicrobials peptides, calmodulin, crinotoxins, pheromone, cytokines, acute-phase proteins, carbonic anhydrase, hemolysin, serotransferrin, heat shock proteins, superoxide dismutase, and pentraxins. Cortisol, glucose, lactic acids, reactive oxygen species, and cellular antioxidants were also detected in the epidermal mucus and have the potential as stress biomarkers. Aside from its potential as a biological matrix to assess the immunity and health status of fish, epidermal mucus also serves as an ecotoxicological biomonitoring tool by detecting biochemical biomarkers responses that arise. We encourage future studies to assess the potentials of the epidermal mucus biological activity using the proteomics approach, given the diversity of fish species. Knowledge about fish health and welfare is important for the conservation and preservation of species biodiversity.

\section{INTRODUCTION}

Stress and disease in fish often occur in the aquaculture industry and the aquatic environment. Microbial infection, water quality, and improper management are factors that may cause stress and disease in fish (Guardiola et al., 2016). Fish live in an environment surrounded by microbes. Its skin is susceptible to infection because of 
contact and exposure to water microorganisms. The decrease in water quality occurs because of changes in dissolved oxygen, water $\mathrm{pH}$, carbon dioxide, temperature, salinity, photoperiod (Cabillon and Lazado, 2019), ammonia pollution (Al-Zaidan et al., 2013) and heavy metals pollution (Omidi et al., 2020). Stress caused by management factors includes crowding, nutritional deficiencies, and improper handling in sorting, storage, or transportation, resulting in adverse effects on fish physiology (Albaladejo-riad et al., 2020). Stress can reduce the body's immune system, reducing resistance to pathogenic invasion and affecting the survival of fish, which makes it susceptible to disease. Fish has a complex defence system mechanism to ward off stress and disease, one of which is the mucous layer as a mucosal defence system that serves as (i) a stable physical and biochemical barrier against pathogenic invasion and environmental pressures such as exposure to pollutants, (ii) coatings that protect epithelial cells in gills, skin, and gastrointestinal tissues. The bioactivities of the mucous layer are a key mechanism in the innate immune system in fish. Weak mucus activities in the first line of defence resulted in disease.

Pressure on the aquatic environment because of anthropogenic factors such as pollution also results in oxidative stress, therefore fish must have a strong host defence system. Fish skin is susceptible to pollutants and is the target of oxidative stress, so it requires routine ecotoxicological biomonitoring. Monitoring of water pollution in the last decade have used epidermal mucus as a potential biological matrix to analyze oxidative stress biomarkers with non-invasive methods for early detection of stress responses and for tracing the effects of pollutants in fish (Omidi et al., 2020; Montenegro et al., 2020). Using skin mucus as a biological matrix in monitoring fish health status is in line with the principle of environmental conservation because, in its analysis, it does not lead to stress, wounds, or death in fish, especially in endemic and protected rare fish. The epidermal mucus has also been used as early detection in monitoring fish health status in the aquaculture industry against pathogenic invasion, especially bacteria, viruses, and parasites. Mucus with mucins as its key components is a slimy, slippery layer, covering the epidermal surface, in the form of viscous colloids containing antimicrobial bioactive compounds, proteins, lipids and water. Epidermal mucus serves as an important component of the innate immune system mechanism in two ways, first by producing mucus continuously to form antibiofilm agents and releasing them regularly. This prevents the pathogens from invading again or as an anti-adhesion to the formation of stable colonization by potentially infectious microbes and blocking parasitic invasion (Dash et al., 2018; Patel et al., 2020). Secondly, epidermal mucus has also been shown to have antimicrobial activity mediated through a series of innate immune factors such as lysozyme, immunoglobulin, complement proteins, lectins, C-reactive proteins, proteolytic enzymes, proteases, alkaline phosphatase, antibacterial proteins, and antimicrobial peptides (Dash et al., 2018; Omidi et al., 2020; Ghalambor et al., 2020). Antimicrobial peptides secreted by immune cells and released into mucus are piscidin, epinecidin-1, and chrysophsins (Fekih-Zaghbib et al., 2013). Other bioactive components of epidermal mucus are cytokines, acute-phase proteins, carbonic anhydrase, and hemolysin (Dang et $a l ., 2020)$. Therefore, fish relies heavily on its innate immune system because its adaptive immune system is relatively underdeveloped (Ángeles Esteban, 2012). Besides, cortisol, glucose, and lactic acid detected in epidermal mucus have the potential to be stress biomarkers. (Guardiola et al., 2016; Fernández-Montero et al., 2020; Ouyang et al., 
2020). Other biomarker responses are the excessive production of reactive oxygen compounds and the detection of cellular antioxidants in epidermal mucus, which also acts as an early detection method of oxidative stress in fish (Dzul-Caamal et al., 2016a; 2016b).

This paper reviews (i) fish's skin mucus components and their bioactivities in response to stress due to pathogenic invasion and environmental pressure; (ii) biochemical responses detected in fish skin mucus as stress biomarkers. Biomarkers in mucus have the potential as a non-invasive method for early detection in evaluating fish stress and health status. We expect the use of mucus biochemical responses as biomarkers to be increasingly popular to assess sub-lethal effects in fish. Thorough understanding of the biological activity of fish skin mucus components and biochemical responses to stress-related mucus will provide thorough knowledge on the mechanisms of the fish's mucosal immune system that are useful for (i) assessing fish health status to develop health management in the modern aquaculture industry and ecotoxicological biomonitoring studies; (ii) facilitate the development of new vaccination strategies and the development of therapeutic applications in fish.

\section{REVIEW}

\section{Epidermal mucus components}

Teleost fish mucus is similar to mammal mucus, which mainly composed of mucins (Shephard, 1993). Mucins are produced by goblet cells, club cells, and sacciform cells in the fish epithelium (Reverter et al., 2018; Xiong et al., 2020). Sticky gel-shaped mucin, insoluble in water, comprising glycoproteins containing high-molecular-weight oligosaccharides (Gobi et al., 2018). Mucins determine the viscoelasticity and rheology of epidermal mucus (Ángeles Esteban, 2012). Mucins serve as a mechanical barrier that filters and prevents pathogenic invasion against the underlying tissue. Epidermal mucus is known as an important element in the mucosal barrier and immune system of fish (Dang et al., 2020). The bioactivity of the epidermal mucus as an antimicrobial depends on the function of its constituent components, namely proteins, carbohydrates, lipids, water, and metabolites (Dash et al., 2018). Fish skin mucus contains different fatty acids, namely saturated fatty acids, monosaturated fatty acids, and polyunsaturated fatty acids. The saturated fatty acids in epidermal mucus are palmitic acids and stearic acids. Monosaturated fatty acid is oleic acid. The polyunsaturated fatty acids are linoleic, alphalinolenic, and moroctic acid. These fatty acids serve as a defence against pathogenic invasion (Dash et al., 2018). Fish skin mucus is also reported to contain several metabolites that have antibacterial properties such as azelaic acid, N-acetylneuraminic acid and $\mathrm{N}$-acetylglucosamine, and hydroxyisocaproic acid (Ekman et al., 2015).

Some researchers have identified the bioactive components of epidermal mucus, i.e. high-molecular-weight glycoproteins ( $106 \mathrm{kDa}$ ) (Subramanian et al., 2008), lysozyme (Abolfathi et al., 2020), alkaline phosphatase (Guardiola et al., 2014; Dash et al., 2018), immunoglobulin (Salinas, 2015), complement proteins (Salinas, 2015; Magnadóttir et al., 2019), lectins (Cordero et al., 2016), agglutinin, interferon, vitellogenin (Gobi et al., 2018), proteolytic enzymes, various types of proteases including trypsins, metalloproteases and cathepsin (Dash et al., 2018) and some antimicrobial proteins and antimicrobial peptides that protect fish against pathogenic invasion (Abolfathi et al., 
2020). Epidermal mucus also contains calmodulin (Patel and Brinchmann, 2017), crinotoxins (Reverter et al., 2018), pheromone (Bulloch et al., 2020), cytokines, acutephase proteins, carbonic anhydrase, hemolysin (Dang et al., 2020), serotransferrin, heat shock proteins, superoxide dismutase (Xiong et al., 2020), and pentraxins (Magnadóttir et al., 2019).

\section{Epidermal mucus bioactivities as a stress response}

Mucus covers the skin and gills of fish as the first surface layer. It always in contact with the aquatic environment so that the emerging biochemical responses are suitable as biomarkers for early detection of stress or disease because of pathogenic invasion and environmental pressures (Dzul-Caamal et al., 2016). Fish skin mucus according to Tarnawska et al. (2019) has been used as stress biomarkers, among others, by detecting antioxidant activities, enzymes (esterases, proteases), non-enzymatic proteins (vitellogenin, radiata zone proteins), hormones (cortisol), and innate immunity. According to Reverter et al. (2018), molecular bioactivities in fish skin mucus serve as an antimicrobial, innate immune system, cellular metabolism, carbohydrate metabolism, lipid metabolism, and protection against ultraviolet exposure. Molecular bioactivities in fish mucus also involved in ecological interactions such as same-sex communication, as cues of finding suitable habitat, partners, or as an alarm signal to danger.

Epidermal mucus serves as a dynamic physical and biochemical protection, and biological and ecological activities such as; communication, sensory perception, locomotion, respiration, ion setting, osmoregulation, excretion, and temperature setting (Ángeles Esteban, 2012), protection against friction, protection against environmental toxins and heavy metal toxicity, parental feeding, and protection against pathogens (DzulCaamal et al., 2016; Reverter et al., 2018). Epidermal mucus is dynamic and semipermeable which allows the exchange of nutrients, water, gas, aroma, hormones, and gametes. Epidermal mucus also acts as a biological barrier because its component activities involved in the immune response can trap or immobilize pathogens so they cannot penetrate the epithelial layer of the epidermis (Cone, 2009; Ángeles Esteban, 2012; Gobi et al., 2018). Stressed fish due to exposure to chemical pollutants such as heavy metals, secreted more mucus as a barrier, inhibiting the diffusion of chemicals. Mucus can bind organic and inorganic materials and eliminate those materials by expelling them into the environment. Consequently, chronic toxin exposures to the mucosal epithelium layer decrease the number of mucosal cells and the thickness of the epithelium layer (Dang et al., 2019). The mucosal barrier in the skin, gills, and intestines of fish is essential as an ecotoxicological biomonitoring tool.

The composition and characteristics of epidermal mucus such as rheological and viscoelasticity properties are essential to supporting their functional activities in the innate immune system (Lai et al., 2009). The content of bioactive compounds and fish mucus characteristics varies depending on fish species, endogenous factors such as gender and developmental stage, and exogenous factors such as stress, hyperosmolarity, temperature, pH, and infection (Ángeles Esteban, 2012). Stress conditions (e.g., crowding, stress handling, nutrient deprivation, ammonia pollution, and exposure to toxic substances) may affect the production and composition of mucus bioactive compounds, disrupt the fish health status and increase its susceptibility to pathogenic bacteria (AlZaidan et al., 2013; Terova et al., 2011; Easy and Ross, 2010; Liu et al., 2013; Reverter et al., 2018). Stressed and injured fish contain a lot of protein in their skin mucus 
(Ángeles Esteban, 2012). Mucus viscoelasticity determines its ability to withstand bacterial motility. Several studies suggest that when stressed, fish will produce mucus continuously (Cone, 2009), increase its mucus secretion and change its mucus composition (van der Marel et al., 2010; Gustafsson et al., 2013; Rajan et al., 2013).

Bioactivities of fish epidermal mucus as a stress response in this review focuses on (i) the bioactive components of the epidermal mucus that play roles in the mucosal immune system, (ii) the bioactive components of the epidermal mucus that function as antimicrobials and (iii) the epidermal mucus as a potential biological matrix to analyze oxidative stress biomarkers due to environmental pressures such as heavy metal pollution and aromatic polycyclic hydrocarbons.

\section{Epidermal mucus bioactivities in innate immunity}

Teleost fish has an active mucosal immune system (Ángeles Esteban, 2012; Salinas, 2015). The mucosal immune system is composed of innate and adaptive immune cells and molecules that work together to protect the host from pathogenic invasion (Guardiola et al., 2016). Thus, fish has a vital component in the defence system which is mucosa-associated lymphoid tissue (MALT). MALT found in the skin is called skinassociated lymphoid tissue (SALT) in the gills called gill-associated lymphoid tissue (GIALT), in the nasopharynx is called nasal-associated lymphoid tissue (NALT) and in the digestive tract is called gut-associated lymphoid tissue (GALT) (Salinas, 2015; Guardiola et al., 2016; Cabillon and Lazado, 2019). Thus, fish skin contains SALT and various types of leukocytes such as lymphocytes, granulocytes, and macrophages, which quickly migrate to the site of infection to kill pathogens (Xiong et al., 2020). Other cellular components that contribute to the fish's innate immunity are mast cells and mucosal dendritic cells (Reverter et al., 2018).

Lately, epidermal mucus is used as a source for proteomic mapping and the discovery of new protein molecules involved in mucosal immunity (Cordero et al., 2016; Fæste et al., 2020; Xiong et al. 2020). A metabolomic approach to fish skin mucus has been used to monitor the health status of fathead minnow (Pimephales promelas) by detecting 204 different metabolites (Ekman et al., 2015). Meanwhile, Fæste et al. (2020) with the proteomics approach has succeeded in identifying 1192 proteins from Atlantic salmon skin mucus. A total of 918 proteins has been identified by Xiong et al. (2020) of 54443 spectra which refers to the yellow catfish (Pelteobagrus fulvidraco) genome database. The proteomics approach has identified that proteins such as lectins, complement proteins, antimicrobial peptides, and immunoglobulins play roles in innate immunity. Proteomics data provide information about protein profiles to comprehensively understand the function and biological activity of fish skin mucus in fighting microbial infections. These proteins are detected in mucus, so it is useful for evaluating the stress and health status of fish (Fæste et al., 2020; Xiong et al., 2020; Dang et al. 2020). Epidermal mucus is also a source for analyzing changes in a humoral immune activity such as immunoglobulin, lysozyme, or alkaline phosphatase (Cordero et al., 2016). Mucosal immunity in the epidermal mucus of fish depends on ecological and physiological conditions such as seasonal cycles and developmental stages. For example, lysozyme levels are affected by the seasonal cycles. In the season when the risk of contracting diseases are high, lysozyme levels in mucus and blood will increase. The stages of immune system development show that the younger the level of immune 
maturity, the lower the ability to kill pathogens. The integrity of epidermal mucus is very essential for the welfare and survival of fish (Abolfathi et al., 2020).

The bioactivities of epidermal mucus in innate immunity depend on its protein components such as lysozyme, phosphatase, esterases, proteolytic enzymes, complement proteins, lectins, immunoglobulins, and C-reactive proteins. The activities of these protein components seek to eliminate pathogens and produce immunity when infection occurs (Reverter et al. 2018; Ghalambor et al., 2020). Lysozymes, alkaline phosphatases, and proteases have acted as hydrolytic enzymes in rainbow trout skin mucus (Abolfathi et al., 2020). Lysozyme is an enzyme that breaks the bond of $\beta$-1,4-glycosides between $\mathrm{N}$ acetyl glucosamine-acid and muramic $\mathrm{N}$-acetyl acid in peptidoglycan, so it can damage the cell walls of bacteria in hypoosmotic environmental conditions (Dash et al., 2018; Abolfathi et al., 2020; Srichaiyo et al., 2020). Lysozyme found in epidermal mucus plays a role in the mucosal defence mechanism against bacterial, viral and parasitic infections (Guardiola et al., 2014; Ghalambor et al., 2020; Abbas et al., 2020). Estimation of lysozyme levels is diagnostic to determine fish disease status (Abbas et al., 2020). Acid and alkaline phosphatases and esterases are important enzymes in fish epidermal mucus, which have antibacterial agents and potential indicators of stress (Ross et al., 2000; Nigam et al., 2014). The activity of the alkaline phosphatase enzymes in the epidermal mucus of fish is an antibacterial agent and helps to heal wounds (Easy and Ross, 2010). Protease is a catalytic enzyme to hydrolyze protein peptide bonds. Fish epidermal mucus contains various proteases that play a role in the innate immune system mechanism. Proteases eliminate the function of pathogens, and other foreign substances (Dash et al., 2018; Abolfathi et al., 2020). Various types of protease enzymes detected in epidermal mucus are trypsins, serin, metalloproteases, cathepsin, and aminopeptidases (Abolfathi et al., 2020), with serin and metalloproteases being the most dominant (Ángeles Esteban, 2012). Proteases are expressed in response to various immune stimulants, including injuries and pathogenic bacteria invasion (Albaladejo-riad et al., 2020). Lectins are a protein that binds to carbohydrates expressed on the cell surface and can clump pathogenic cells that live in the epidermal mucus. They can coagulate bacteria on the surface of mucous cells by recognizing specific sites on glycoproteins and glycolipids or polysaccharides in bacteria. In the epidermal mucus, lectins play active roles in the mucosal defence system with its activity on the external surface of the body (Dash et al., 2018). Some complementary proteins such as C7, C3, and C1q have been identified in the epidermal and intestinal mucus of several fish species (Shen et al., 2012; Fan et al., 2015; Salinas, 2015; Reverter et al., 2018). Immunoglobulins (IgM and IgT/IgZ) are the key components of innate immunity in the epidermal mucus, with IgT/IgZ having the primary activity in fish mucosal immunity (Xu et al., 2013; Sunyer, 2013; Xia et al., 2016; Reverter et al., 2018; Pietrzak et al., 2020). C-reactive proteins are protein molecules in epidermal mucus extracellular vesicles that play an important role in innate immune defence. C-reactive proteins are formed as a reaction from infection, injury, and inflammatory processes. High levels of C-reactive proteins show inflammation in the body. C-reactive proteins play roles in repairing damaged tissues (Easy and Ross, 2010). Stressed fish have higher C-reactive protein levels than normal (Magnadóttir et al., 2019).

Other molecules involved in the innate immune activity are glycoproteins such as transferrin (Easy et al., 2012). Transferrin is an iron-binding glycoprotein that plays a role in iron transportation (absorption, storage, disposal) in fish epidermal mucus. 
Therefore, transferrin plays an important role in the innate defence mechanism by binding to iron and reducing its availability from pathogens by chelating it. Transferrin's activity help resists pathogenic growth until the emergence of the immune system response (Dash et al., 2018). Catfish mucus contains mucins whose major component is glycoproteins. Mucins in mucus serve (i) to coat the surface of epithelial tissue, (ii) as a lubricant and protector, (iii) prevents parasitic, bacterial and fungal colonization, (iv) becomes the first line of defence against water friction and pathogenic invasion (Abdel-Shafi et al., 2019). Fish epidermal mucus also contains antibacterial peptides that function to modulate the function of B lymphocyte cells that play an essential role in the innate immune system (Reverter et al., 2018). Epidermal mucus and its components from various fish species can be a source of new antimicrobial agents and are involved in the innate immune system in fish (Table 1).

Table 1. Summary of epidermal mucus bioactivity as a tool of evaluating the health status of several fish species

\begin{tabular}{|c|c|c|c|c|}
\hline Bioactivity & $\begin{array}{l}\text { Bioactive molecules are involved in } \\
\text { immune and/or stress responses }\end{array}$ & Stressors & Source organism & References \\
\hline \multirow[t]{2}{*}{ Mucosal barrier } & Lectin, alkaline phosphatase, lysozyme & $\begin{array}{l}\text { Parasitic } \\
\text { infection }\end{array}$ & $\begin{array}{l}\text { Ctenophryngodon idella, } \\
\text { Hypophthalmichthys } \\
\text { moiltrix, Labeo rohita, } \\
\text { Cirrhinus mrigala, Catla } \\
\text { catla, Cyprinus carpio }\end{array}$ & $\begin{array}{l}\text { Abbas et al. } \\
(2020)\end{array}$ \\
\hline & Sialic acid (N-acetylneuraminic acid) & Exposed to zinc & $\begin{array}{l}\text { Rainbow trout (Salmo } \\
\text { gairdneri RICHARDSON) }\end{array}$ & $\begin{array}{l}\text { Eddy \& Fraser } \\
\text { (1982) }\end{array}$ \\
\hline \multirow[t]{13}{*}{$\begin{array}{l}\text { Antibacterial } \\
\text { activity }\end{array}$} & Glycoprotein & Bacteria infection & $\begin{array}{l}\text { African catfish } \\
\text { (Clarias gariepinus) }\end{array}$ & $\begin{array}{l}\text { Abdel-Shafi et } \\
\text { al. (2019) }\end{array}$ \\
\hline & Peroxidase, IgM, protease & Feed deprivation & $\begin{array}{l}\text { Gilthead Seabream } \\
\text { (Sparus aurata) }\end{array}$ & $\begin{array}{l}\text { Albaladejo-riad } \\
\text { et al. }(2020)\end{array}$ \\
\hline & Antibacterial Peptides & Escherichia coli & $\begin{array}{l}\text { Yellow Fish Skin } \\
\text { (Pelteobagrus fulvidraco) }\end{array}$ & $\begin{array}{l}\text { Fakih \& Dewi } \\
(2020)\end{array}$ \\
\hline & NK-lysin & $\begin{array}{l}\text { Aeromonas } \\
\text { salmonicida, } \\
\text { Vibrio } \\
\text { anguillarum }\end{array}$ & Salmo salar & $\begin{array}{l}\text { Valero et al. } \\
\text { (2019) }\end{array}$ \\
\hline & Complement C1qC & Escherichia coli. & $\begin{array}{l}\text { Siberian sturgeon } \\
\text { (Acipenser baerii) }\end{array}$ & $\begin{array}{l}\text { Fan et al. } \\
(2015)\end{array}$ \\
\hline & Complement C7 & $\begin{array}{l}\text { Aeromonas } \\
\text { hydrophila }\end{array}$ & Grass carp & $\begin{array}{l}\text { Shen et al. } \\
\text { (2012) }\end{array}$ \\
\hline & The antimicrobial peptide, Chrysophsins & - & Dicentrarchus labrax & $\begin{array}{l}\text { Fekih-Zaghbib } \\
\text { et al. }(2013)\end{array}$ \\
\hline & Glycoproteins & - & $\begin{array}{l}\text { Mudskipper } \\
\text { (Boleophthalmus } \\
\text { pectinirostris) }\end{array}$ & $\begin{array}{l}\text { H. han Liu et } \\
\text { al. }(2019)\end{array}$ \\
\hline & Proteinaceous compounds & - & $\begin{array}{l}\text { Mudskipper } \\
\text { Periophthalmodon } \\
\text { schlosseri (Pallas, 1770) }\end{array}$ & $\begin{array}{l}\text { Mahadevan et } \\
\text { al. (2019) }\end{array}$ \\
\hline & Antimicrobial peptides & $\begin{array}{l}\text { B. subtilis, } S . \\
\text { aureus, } \text { E. coli, } \\
P . \text { aeruginosa }\end{array}$ & Puntius sophore & $\begin{array}{l}\text { M. Patel et al. } \\
(2020)\end{array}$ \\
\hline & Antimicrobial polypeptides, piscidins & Acute stress & $\begin{array}{l}\text { Marine sea bass } \\
\text { (Dicentrarchus labrax) }\end{array}$ & $\begin{array}{l}\text { Terova } \text { et al. } \\
\text { (2011) }\end{array}$ \\
\hline & $\begin{array}{l}\text { Lysozyme, protease, alkaline phosphatase, } \\
\text { esterase }\end{array}$ & Yersinia ruckeri & $\begin{array}{l}\text { Rainbow trout } \\
\text { (Oncorhynchus mykiss) }\end{array}$ & $\begin{array}{l}\text { Sheikhzadeh } \text { et } \\
\text { al. (2012) }\end{array}$ \\
\hline & Peptides & $\begin{array}{l}\text { Salmonella } \\
\text { enterica C610 }\end{array}$ & $\begin{array}{l}\text { Arctic char (Salvelinus } \\
\text { alpinus), brook trout ( } S \text {. } \\
\text { fontinalis), koi carp } \\
\text { (Cyprinus carpio sub sp. }\end{array}$ & $\begin{array}{l}\text { Subramanian et } \\
\text { al. (2008) }\end{array}$ \\
\hline
\end{tabular}


Grammistin

Mucosal cellular innate immunity
Lysozyme, alkaline phosphatase proteases

Peroxidase, IgM, protease

Terminal carbohydrates, protease, antiprotease, peroxidase, lysozyme, IgM Lysozyme, peroxidase

C-Reactive protein, lysozyme

Trypsin

Peroxidase

Alkaline phosphatase, cathepsin B, lysozyme, cortisol

Transferrin

Lysozyme

Lysozyme

Protein, alkaline phosphatase, myeloperoxidase, lysozyme

Terminal carbohydrates

(N-acetylneuraminic acid, mannose, glucose and $\mathrm{N}$-acetylgalactosamine), proteases, lysozyme, peroxidase, alkaline phosphatase, esterases, ceruloplasmin Protease, peroxidase

Lectin, terminal carbohydrate

Peroxidase

Alkaline phosphatase, lysozyme, protease, lectin

Alkaline phosphatase

Glycoprotein

\section{Lysozyme}

Peptidylarginine deiminase, complement component C3, C-reactive proteins Lysozyme, protease, alkaline phosphatase, esterase, transferrin, IgM koi), striped bass (Morone saxatilis), haddock

(Melanogrammus aeglefinus), hagfish

(Myxine glutinosa)

Soapfish

(Grammistes sexlineatus)

Effects of season

Rainbow trout,

(Oncorhynchus mykiss)

Sugiyama et al. (2005)

Abolfathi et al. (2020)

Feed deprivation

Gilthead Seabream

(Sparus aurata)

Skin ulcers

Gilthead seabream

(Sparus aurata)

Oreochromis niloticus

Sewage

chemicals

Common carp

(Cyprinus carpio L.)

Environmental

influence

Exposed to

cadmium

Short-term stress,

Long-term stress

Smoltification

Atlantic salmon

(Salmo salar L.)

Cyprinus carpio L.

Atlantic salmon

(Salmo salar L.)

Atlantic cod

(Gadus morhua)

Atlantic salmon

Salt

concentration

Aeromonas

hydrophila

infection

Micrococcus

lysodeikticus

infection

(Salmo salar)

Cyprinus carpio

Oreochromis mossambicus

Senegalese sole

(Solea senegalensis, Kaup)

Albaladejo-riad et al. (2020)

Tapia-Paniagua et al. (2018)

Srichaiyo et al. (2020)

Tarnawska $e t$ al. (2019)

Braun et al. (1990)

Brokken et al, (1998)

Easy \& Ross

(2010)

Easy et al. (2012)

Fagan et al. (2003)

Ghalambor et al. (2020)

Gobi et al. (2018)

Guardiola et al. (2017)

Acute crowding, Sparus aurata L.

Guardiola et al. anaesthetic agents, air exposure

Heavy metals

(As, $\mathrm{Cd}, \mathrm{Hg}$ )

Thermal

pollution

Brackishwater

polyculture

Environment, nutrition

Sparus aurata $\mathrm{L}$.

Oncorhynhcus mykiss

Lates calcarifer

Chanos chanos,

Mugil cephalus

Various species of fish

Mudskipper

(Boleophthalmus

pectinirostris)

Short-Term feed Channel catfish deprivation

(Ictalurus punctatus)

Cod (Gadus morhua L.)

Olive flounder

(Paralichthys olivaceus)

ococcus lysodeikticus infection
(2016)

Guardiola et al. (2015)

Iger et al. (1994)

Kumar et al. (2019)

Lallès (2019)

H. han Liu et al. (2019)

L. Liu et al. (2013)

Magnadóttir et al. (2019)

Palaksha et al. (2008) 
Proteomic profile: Calmodulin, cystatin-B, histone $\mathrm{H} 2 \mathrm{~B}$, peroxiredoxin1, apolipoprotein A1, natterin-2, 14-3-3 protein, alfa enolase, pentraxin, warm temperature acclimation $65 \mathrm{kDa}$ (WAP65kDa) and heat shock proteins Acute-phase protein, antimicrobial proteins, cytokines, lectin, lysozymes, mucin, peroxidase, proteases, oxidoreductase

Calpain small subunit 1, glutathione-Stransferase omega 1 , proteasome $26 \mathrm{~S}$ subunit, 14-kDa apolipoprotein, beta 2tubulin, cold-inducible RNA binding protein, malate dehydrogenase 2 (mitochondrial) and type II keratin Protease, cortisol

Actins, keratins, glycolytic enzymes, ubiquitin, heat shock proteins, transferrin, hemopexins

Lysozyme, protease, carboxylesterase, alkaline phosphatase, acid phosphatase, catalase, peroxidase,

Lysozyme, proteases, cathepsin B; alkaline phosphatase

Lysozyme, alkaline phosphatase, esterase, protease, protein

Lysozyme, peroxidase, alternative complement (ACH50)

Lysozyme, protease, anti-protease, cathepsin B, alkaline phosphatase, peroxidase)

\section{$\operatorname{IgZ}$}

Lectins, complement components, antibacterial peptides, immunoglobins, structural proteins

Biomarker of F2-isoprostanes oxidative stress

Metallothioneins, HSP70

$\mathrm{O}_{2} \bullet, \mathrm{H}_{2} \mathrm{O}_{2}$, TBARS (thiobarbituric acid reactive substances), carbonyl proteins, superoxide dismutase (SOD), catalase (CAT), glutathione peroxidase (GPx), vitellogenin (VTG) and metallothionein (MT)
Pathogens and external stressors

Lumpsucker

(Cyclopterus lumpus)

Cyprinus carpio

Vibrio

anguillarum

Atlantic cod

(Gadus morhua)

Salmo salar

Sparus aurata

Indian major carp

(Labeo rohita)

Arctic char (Salvelinus

alpinus), brook trout ( $\mathrm{S}$.

fontinalis), koi carp

(Cyprinus carpio), striped bass (Morone saxatilis), haddock, (Melanogrammus aeglefinus), Atlantic cod (Gadus morhua), hagfish (Myxine glutinosa).

Clarias gariepinus, Channa micropeltes, Channa striatus, Oxyeleotris marmorata, Oreochromis niloticus, Hemibagrus nemurus

Streptococcus agalactiae

Aeromonas

hydrophila,

Vibrio

parahaemolyticus

Aeromonas

hydrophila

Edwardsiella ictaluri

Waste pollution

Sewage

chemicals

Metals and PAHs
Oreochromis niloticus

Amphiprion clarkii

Blunt snout bream

(Megalabrama

amblycephala)

Yellow catfish

(Pelteobagrus fulvidraco)

Esox lucius, Salvelinus namaycush

Common carp (Cyprinus

carpio L.)

Blackfin goodeid

(Girardinichthys viviparus)
Patel \&

Brinchmann (2017)

Pietrzak et al. (2020)

Rajan et al. (2013)

Ross et al. (2000) Sanahuja \& Ibarz (2015)

Srivastava et al. (2018)

Subramanian et al. (2007)

Timalata et al. (2015)

Van Doan et al. (2019)

Wang et al. (2019)

Xia et al. (2016)

Xiong et al. (2020)

Bulloch et al. (2020)

Tarnawska et al. (2019)

Dzul-Caamal et al. (2016a) 


\begin{tabular}{|c|c|c|c|c|}
\hline & $\begin{array}{l}\mathrm{O}_{2} \cdot, \mathrm{H}_{2} \mathrm{O}_{2} \text {, TBARS, carbonyl proteins, } \\
\text { superoxide dismutase (SOD), catalase } \\
(\mathrm{CAT}) \text {, glutathione peroxidase (GPx), } \\
\text { vitellogenin (VTG) and metallothionein } \\
(\mathrm{MT})\end{array}$ & $\begin{array}{l}\text { Exposed to crude } \\
\text { oil }\end{array}$ & Goodea gracilis & $\begin{array}{l}\text { Dzul-Caamal, } \\
\text { et al. }(2016 \mathrm{~b})\end{array}$ \\
\hline & $\begin{array}{l}\text { Reactive oxygen species (ROS), reactive } \\
\text { nitrogen species (RNS), antioxidant } \\
\text { parameters of superoxide } \\
\text { dismutase (SOD), glutathione peroxidase } \\
(\mathrm{GPx})\end{array}$ & $\begin{array}{l}\text { Aeromonas } \\
\text { hydrophila } \\
\text { infection }\end{array}$ & Oreochromis mossambicus & $\begin{array}{l}\text { Gobi et al. } \\
\text { (2018) }\end{array}$ \\
\hline & Carboxylesterase & $\begin{array}{l}\text { Organophosphate } \\
\text { exposure }\end{array}$ & Cirrhinus mrigala & $\begin{array}{l}\text { Nigam et al. } \\
(2014)\end{array}$ \\
\hline & $\begin{array}{l}\text { Total antioxidant capacity, total oxidative } \\
\text { status, esterase, cortisol }\end{array}$ & $\begin{array}{l}\text { Gold } \\
\text { nanoparticles }\end{array}$ & Sparus aurata $\mathrm{L}$. & $\begin{array}{l}\text { Oliveira et al. } \\
\text { (2018) }\end{array}$ \\
\hline $\begin{array}{l}\text { Modulate the } \\
\text { immune system } \\
\text { and bioactive } \\
\text { carrier }\end{array}$ & Protein-based nanoparticles & - & Lophiosilurus alexandri & $\begin{array}{l}\text { Charlie-Silva et } \\
\text { al. (2019) }\end{array}$ \\
\hline \multirow[t]{3}{*}{$\begin{array}{l}\text { Mucosal } \\
\text { humoral } \\
\text { immunity }\end{array}$} & $\operatorname{IgM}$ & $\begin{array}{l}\text { Effect of freezing } \\
\text { and } \\
\text { lyophilization }\end{array}$ & $\begin{array}{l}\text { Gilthead seabream } \\
\text { (Sparus aurata) }\end{array}$ & $\begin{array}{l}\text { Cordero et al. } \\
(2016)\end{array}$ \\
\hline & $\operatorname{IgM}$ & $\begin{array}{l}\text { Vibrio harveyi, } \\
\text { Vibrio } \\
\text { angillarum, } \\
\text { Photobacterium } \\
\text { damselae, } \\
\text { Escherichia coli, } \\
\text { Bacillus subtilis, } \\
\text { Shewanella } \\
\text { putrefaciens) }\end{array}$ & $\begin{array}{l}\text { Gilthead seabream (Sparus } \\
\text { aurata), European sea bass } \\
\text { (Dicentrarchus labrax), shi } \\
\text { drum (Umbrina cirrosa), } \\
\text { common dentex (Dentex } \\
\text { dentex), dusky grouper } \\
\text { (Epinephelus marginatus) }\end{array}$ & $\begin{array}{l}\text { Guardiola et al. } \\
\text { (2014) }\end{array}$ \\
\hline & $\operatorname{IgM}$ & $\begin{array}{l}\text { Acute crowding, } \\
\text { anaesthetic } \\
\text { agents, air } \\
\text { exposure }\end{array}$ & Sparus aurata $\mathrm{L}$. & $\begin{array}{l}\text { Guardiola et al. } \\
\text { (2016) }\end{array}$ \\
\hline \multirow[t]{8}{*}{$\begin{array}{l}\text { Biomarker of } \\
\text { stress }\end{array}$} & Glucose, lactate, protein, cortisol & $\begin{array}{l}\text { Hypoxia and } \\
\text { netting }\end{array}$ & $\begin{array}{l}\text { Meagre (Argyrosomus } \\
\text { regius) }\end{array}$ & $\begin{array}{l}\text { Fernández- } \\
\text { Alacid et al. } \\
\text { (2019) }\end{array}$ \\
\hline & Glucose, lactate, protein, cortisol & $\begin{array}{l}\text { Fish capture, } \\
\text { bacterial } \\
\text { infection, fasting }\end{array}$ & $\begin{array}{l}\text { Meagre, sea bass, sea } \\
\text { bream }\end{array}$ & $\begin{array}{l}\text { Fernández- } \\
\text { Alacid et al. } \\
\text { (2018) }\end{array}$ \\
\hline & Cortisol, $m и с-2$ gene expression & $\begin{array}{l}\text { Handling, } \\
\text { density, } \\
\text { temperature, } \\
\text { fasting }\end{array}$ & $\begin{array}{l}\text { Greater amberjack } \\
\text { (Seriola dumerili) }\end{array}$ & $\begin{array}{l}\text { Fernández- } \\
\text { Montero et al. } \\
\text { (2020) }\end{array}$ \\
\hline & Cortisol & $\begin{array}{l}\text { Acute crowding, } \\
\text { anaesthetic } \\
\text { agents, air } \\
\text { exposure }\end{array}$ & Sparus aurata L. & $\begin{array}{l}\text { Guardiola et al. } \\
\text { (2016) }\end{array}$ \\
\hline & Cholinesterase & $\begin{array}{l}\text { Organophosphate } \\
\text { exposure }\end{array}$ & $\begin{array}{l}\text { Cirrhinus mrigala, Labeo } \\
\text { rohita, Catla catla }\end{array}$ & $\begin{array}{l}\text { Nigam et al. } \\
\text { (2014) }\end{array}$ \\
\hline & $\begin{array}{l}\text { Lysozyme, IgM, alkaline phosphatase, } \\
\text { protein total }\end{array}$ & Lead pollution & Neogobius melanostomus & $\begin{array}{l}\text { Omidi et al. } \\
(2020)\end{array}$ \\
\hline & Cortisol, glucose, lactate & MS-222 exposure & Symphysodon aequifasciata & $\begin{array}{l}\text { Ouyang et al. } \\
(2020)\end{array}$ \\
\hline & Cytokeratins & Chronic stress & Sparus aurata $\mathrm{L}$. & $\begin{array}{l}\text { Pérez-Sánchez } \\
\text { et al. (2017) }\end{array}$ \\
\hline $\begin{array}{l}\text { Biomarker of } \\
\text { endocrine } \\
\text { disruptor }\end{array}$ & Vitellogenin, zona radiata protein & $\begin{array}{l}\text { Waterborne } \\
\text { nonylphenol }\end{array}$ & Salmo salar $\mathrm{L}$. & $\begin{array}{l}\text { Meucci \& } \\
\text { Arukwe (2005) }\end{array}$ \\
\hline
\end{tabular}

\section{Epidermal mucus bioactivity as antimicrobial}


Fish epidermal mucus contains antimicrobial peptides, which is one of the primary molecules to fight pathogenic microbial invasions (Cipolari et al., 2020). Antimicrobial peptides are endogenous peptides with short-chain, cationic, amphipathic, and molecular weight less than $13 \mathrm{kDa}$. Antimicrobial peptides are a component of the innate immune system found on the surface layer of cytolytic and microbicidal epithelial tissues. These peptides generally consist of 10 to 50 amino acids with 2 to 8 positive charges and have various activities, including inhibiting microbial growth of Gram-positive and Gramnegative bacteria, fungi, viruses, and parasites (Lei et al., 2019). Antimicrobial peptides are key components of the innate immune system that acts as the first line of defence against various pathogenic microbial invasion without having high specificity or memory. Other biological activities of antimicrobial peptides are immunocompetence and homeostasis (Chaturvedi et al., 2020), as well as an immunomodulator to enhance body immunity (Gokhale and Satyanarayanajois, 2014). The types of antimicrobial peptides found are (i) $\alpha$-helical peptides piscidins (moronecidins, pleurocidins, dicentracins, and chrysopsins, (ii) linear peptides such as pardaxin and pelteobagrin, (iii) defensin namely cysteine-rich antibacterial peptides, (iv) grammistins (v) cathelicidins (vi) histone-like proteins (Reverter et al. 2018; Cipolari et al., 2020; Chaturvedi et al., 2020). Antimicrobial peptides are derived from biologically inactive proteins that are processed to the active forms and derived from functional proteins (Reverter et al., 2018). The expression of antimicrobial peptides induced by molecular patterns associated with pathogens and molecular patterns associated with damage (Chaturvedi et al., 2020). The mechanism of antimicrobial action of these peptide molecules is done by crossing the cell membrane, interacting with microbial DNA, thus disrupting transcription and/or replication (Dash et al., 2018; Chaturvedi et al., 2020). Peptides in the epidermal mucus of Clarias gariepinus have functioned as an antibacterial by preventing the colonization of Gram-positive and Gram-negative pathogens. Peptides also contain secretin/glucagon neuropeptides that have an immune response to acute bacterial infections (Abdel-Shafi et al., 2019). Other antibacterial proteins found in fish mucus are L-amino acid oxidases, ribosomal proteins such as L40, L36A, L35, and S30, and protein such as haemoglobin (Hb- $\beta$ ) (Reverter et al., 2018).

Commensalism microbiota in fish epidermal mucus plays an important role in controlling opportunistic pathogens. Recent studies have shown specific mechanisms by which the host can recognize commensal bacteria, and their metabolites can control the proliferation of pathogenic bacteria. For example, Reverter et al. (2018) explain that the bacterium Flectobacillus major from the mucous layer of the rainbow trout skin produces sphingolipids which induce the production of immunoglobulin $\mathrm{T}(\operatorname{IgT})$, forming $\mathrm{B}$ lymphocyte cells and antibody responses, to control the growth of pathogenic bacteria. Another study found special metabolites that have antibacterial and antifungal activities in bacterial strains isolated from fish mucus, so it can control the development of host pathogenic bacteria.

\section{Biochemical biomarkers in fish epidermal mucus as a stress response}

Stress can decrease the immune system, reducing resistance to pathogenic invasions, and threatening the survival of fish. In stressful situations, there is a relationship between the endocrine system and the immune system, e.g. cytokines and neuropeptide role in the neuroendocrine and immune systems. Thus, the study of the interaction between the endocrine system and the immune system in fish mucosa, after its 
exposure to stress, continues to develop as an alternative in non-invasive early detection efforts (Guardiola et al., 2016).

The fish mucosal immune system comprises innate and adaptive immune cells that are arranged exclusively and work together to protect the host against pathogenic invasions. One of them is SALT. The skin as the first line of defence can respond to stress agents after activation of receptors on epidermal cells. The epidermal mucus on fish skin acts as a storage/reservoir of various immune components that block various kinds of chemical, physical, and biological stressors (Easy and Ross, 2010; Guardiola et al., 2016). Stressor agents also trigger the production of steroid hormones (e.g. cortisol) which are detected in epidermal mucus as biochemical biomarkers. Primary, secondary, and tertiary effects on stress-affected fish have the potential as biochemical biomarkers. The primary effect that occurs is elevated levels of plasma cortisol and catecholamine. Elevated levels of plasma glucose and lactic acid, respiratory stimulation, and increased oxygen intake are the secondary effects, whereas decreased growth, decreased reproduction, and immunosuppression are the tertiary effects of stress-affected fish. Peroxidase enzyme activity occurs as a non-specific stress response in fish exposed to cadmium (Brokken et al., 1998). The stress on Salmo salar L. because of Lepeophtheirus salmonis infection leads to the release of innate immune factors (protease enzymes, actin proteins, transferrin) and cortisol into the serum and the epidermal mucus to eliminate the effects of stressors (Easy and Ross, 2010). According to Easy and Ross (2010) there are changes in the activity of the alkaline phosphatase, cathepsin B, lysozyme and protease enzymes in Atlantic salmon (Salmo salar L.) mucus due to long-term stress handler and there is a positive correlation between the profile of the enzyme with the hormone cortisol. Guardiola et al. (2016) have examined the levels of epidermal mucus cortisol of Sparus aurata L. as a reliable non-invasive acute-stress biomarker. Besides cortisol, immunoglobulin M, protease enzymes activity and peroxidase enzymes are elevated levels in the stress-affected fish epidermal mucus. Cortisol, produced by the adrenal glands are lipophilic, which can diffuse through the cell membranes to reach several target tissues including secreted into the epidermal mucus. Therefore, measurement of cortisol levels as a biochemical biomarker in epidermal mucus is a non-invasive method to test stress or health status of fish. The study contributes a better understanding of the potential of epidermal mucus as a biochemical biomarker for early detection of stress.

Epidermal mucus of stress-affected fish due to hypoxia and crowding contain structural protein molecules (glycoprotein, lectin, actin, transferrin) and enzymes that serve as the body's defence and metabolism system (Fernández-Alacid et al., 2019). Mucus contains glucose metabolites and cortisol hormone (Guardiola et al., 2016) which positively correlated with plasma cortisol levels, thus potentially as a biochemical biomarker with a non-invasive, fast, and simple method (Fernández-Alacid et al., 2018) to detect early stress response and disease (Fernández-Alacid et al., 2019). Other metabolites found in stress-affected fish mucus are lactic acid and proteins. Lactic acid levels and the ratio of glucose to proteins can be used as stress biomarkers due to environmental stress (Fernández-Alacid et al., 2018).

Fish skin is the key target of oxidative stress because of the production of Reactive Oxygen Species (ROS) resulting from heavy metal pollution and polycyclic aromatic hydrocarbons in aquatic ecosystems. We believe oxidative stress to be a pathway for the mechanism of toxicity in fish exposed to environmental contaminants. Epidermal mucus 
is a biological matrix suitable for analyzing biochemical responses that occur because of oxidative stress. For the first time, Dzul-Caamal et al. (2016) have proven the benefits of the epidermal mucous layer as a bio-indicator of oxidative stress using biochemical biomarker panels such as $\mathrm{O}^{\circ}, \mathrm{H}_{2} \mathrm{O}_{2}$, lipid peroxidation (as TBARS), carbonyl proteins $(\mathrm{RC}=\mathrm{O})$, superoxide dismutase (SOD), catalase $(\mathrm{CAT})$, glutathione peroxidase (GPx), vitellogenin (VTG) and metallothionein (MT) to monitor the health status of protected wild fish namely Girardinichthys viviparus in two polluted lakes in the Mexican Valley. The biochemical biomarker panel is also used to monitor the health of the rare Goodea gracilis fish (Hubbs and Turner, 1939) exposed to polycyclic aromatic hydrocarbons from crude oil spills (Dzul-Caamal et al., 2016). Exposure to these pollutants induces the production of reactive oxygen compounds and elicits antioxidant defence responses detected in the epidermal mucous layer. For the first time, the results of this research have proven the benefits of epidermal mucus as an ecotoxicological biomonitoring tool for monitoring the health status of endemic fish. F2-isoprostanes (F2-isoPs) is also a reliable biochemical biomarker for oxidative stress responses in Salvelinus namaycush exposed to heavy metal pollutants. The levels of these compounds are measured in the liver and plasma, which could kill the fish, but because of social pressure and environmental conservation, a non-invasive method using fish epidermal mucus is used to analyze oxidative stress biomarkers (Bulloch et al., 2020). Fernández-Montero et al. (2020) stated that there is a positive correlation between plasma cortisol levels and cortisol levels in the epidermal mucus of Seriola dumili that were stressed due to temperature factors, stress management, and fasting. тис-2 gene expression occurs as a biomarker of epidermal mucus production.

\section{Prospects of epidermal mucus as a fish health evaluation tool}

The study of fish mucus as a potential biological matrix to evaluate stress and health status of fish due to pathogen invasion and environmental stress has lately increased. Potential fish mucus to be developed as a fish health evaluation tool and bioactive components in the mucus is very diverse so that it has the potential as a natural medicinal ingredient. Several studies in Indonesia that have been conducted are the application of immunocytochemical Streptavidin Biotin (SB) test on the epidermal mucus of the tiger grouper (Epinephelus fuscoguttatus) as an early diagnostic test for viral nervous necrosis (VNN) disease. The fast and accurate SB test is suitable to be applied in routine biomonitoring and VNN prevention programs in the Indonesian Fish Quarantine because it can be done without killing the fish, scientifically accepted, and does not pollute the environment (Sudaryatma et al., 2012). Epidermal mucus of striped snakehead (Channa striata) in gel formulation is used to treat burns in rabbits (Oryctolagus cuniculus) (Safaruddin et al., 2019; Rusli and Yeniati, 2019). Epidermal mucus of Indonesian shortfin eel (Anguilla bicolor) is also a potential candidate for healing burns (Abi et al., 2019). However, protein molecules and their profile which acts as wound healing factors have not been studied. The study of Fakih and Dewi (2020) with In silico computational method successfully simulates the potential of epidermal mucus as a source of antimicrobial peptides, which serves as the first line of defence in humans against pathogenic bacteria, such as Escherichia coli. Some antimicrobial peptides isolated from the skin mucus of yellow catfish (Pelteobagrus fulvidraco) can inhibit Penicillin-Binding Protein 3 (PBP3) in Escherichia coli, including Pelteobagrin, Myxinidin, Pleurocidin, and Pardaxin-P1. These antimicrobial peptides can be selected as 
a natural antimicrobial candidate, even though the study is modelling. In Indonesia, studies on the detection and isolation of antimicrobial peptides, structural proteins and enzymes from fish mucus are still scarce, because of the lack of concern to its antimicrobial potential and its involvement in the mucosal defence system.

The prospect of proteomics studies on the biological epidermal mucus activity needs to be encouraged, given the diversity of fish biodiversity that has the potential to produce bioactive components and metabolites that are involved in the mucosal and antimicrobial immune systems specifically. Research with the proteomics approach is expected to successfully identify new proteins that play a role in innate immunity. Previous studies have not known about protein profiles, so knowledge about its bioactivity is very limited. Proteomics data provide information about protein profiles, which will bring a comprehensive understanding of the function and the biological activity of fish skin mucus in fighting stress and microbial infections. Proteins identified in the mucus are useful for fish stress and health status (Fæste et al., 2020; Xiong et al., 2020; Dang et al. 2020). Opportunities for future studies are wide open to develop the potential of fish mucus as a candidate for natural antimicrobials and stress biomarkers. Study collaboration opportunities focus on proteomics studies aimed at discovering new bioactive protein molecules and their profiles isolated from native Indonesian fish. It's because the potential of fish is not only as a source of nutrients, but also as a source of structural proteins and enzymes involved in the mucosal immune system, a source of antimicrobial peptides, and a source of anti-inflammatory. Antimicrobial peptides derived from fish mucus have broad-spectrum bioactivities, which play roles in cellular communication, as modulators, mediators, hormones, effectors, cofactors, activators, and stimulators. Recent studies on the pharmacological effects of fish peptides show antihypertensive, immunomodulatory, antioxidant, antitumor, and antimicrobial activities, resulting in potentially extra ingredients for diagnostics, therapy, and treatment for humans and animals (Cipolari et al., 2020; Fæste et al., 2020). Besides studies on proteomics, the studies of the influence of variations in environmental factors on the production and function of mucus are crucial to its function as a biological barrier and its role in mucosal immunity. According to Cabillon and Lazado (2019), changes in environmental factors, not only affect the phenotype of the mucous layer structures and molecular responses but also affect the bioactive components of the mucus and the structure of the microbiota lining the surface of the mucosa. It's important to consider the effect of various environmental factors on adaptative mechanisms in the production of mucus. A comprehensive understanding of mucosal structures and long-term adaptation mechanisms in response to environmental changes is indispensable in modern aquaculture production technology with strict control over environmental changes for increased biosecurity and sustainable production systems.

\section{CONCLUSION}

Fish epidermal mucus contains bioactive components such as structural proteins, enzymes, antimicrobial proteins and peptides that have the potentials (i) as antibacterial, antiviral, antifungal and antiparasitic agents and the potential to be developed as new materials for diagnostic, therapeutic or treatment agents in humans and animals; (ii) as the development of non-invasive, non-lethal methods for early detection of infections and 
stress; (iii) as a biological matrix for analyzing biomarkers for oxidative stress because of environmental pollutants. Not only is epidermal mucus a potential biological matrix for assessing fish's immune and health status, but it also acts as a bio-indicator of stress by detecting biochemical biomarkers that emerge.

\section{REFERENCES}

Abbas, F.; Hafeez-ur-Rehman, M.; Ashraf, M.; Iqbal, K.; Andleeb, S.; and Khan, B. (2020). Mucus properties of Chinese carp and Indian carp: Physical barrier to pathogens. Iran. J. Fish. Sci., 193(3): 1221-1236. https://doi.org/10.22092/ijfs.2019.119394.0

Abdel-Shafi, S.; Osman, A.; Al-Mohammadi, A.-R.; Enan, G.; Kamal, N.; and Sitohy, M. (2019). Biochemical, biological characteristics and antibacterial activity of glycoprotein extracted from the epidermal mucus of African catfish (Clarias gariepinus). Int. J. Biol. Macromol., 138: 773 - 780. https://doi.org/10.1016/j.ijbiomac.2019.07.150

Abi, A.; Fadila, V.; Mutmainah, S.; and Fauzi, Y. (2019). Formulation of Eel fish mucus gel (Anguila bicolor) as a candidate for burn healing. J. Pharmaqueous, $106-112$.

Abolfathi, M.; Akbarzadeh, A.; Hajimoradloo, A.; and Joshaghani, H. R. (2020). Seasonal changes of hydrolytic enzyme activities in the skin mucus of rainbow trout, Oncorhynchus mykiss at different body sizes. Dev. Comp. Immunol., 103: 103499. https://doi.org/10.1016/j.dci.2019.103499

Al-Zaidan, A. S.; Endo, M.; Maita, M.; Gonçalves, A. T.; Futami, K.; and Katagiri, T. (2013). A toxicity bioassay study concerning the effect of un-ionized ammonia on the mucus cells response originating from the gills of zebrafish Danio rerio. Fish Sci., 79(1): 129 - 142. https://doi.org/10.1007/s12562-012-0573-6

Albaladejo-riad, N., Espinosa-ruíz, C., and Esteban, M. Á. (2020). Feed deprivation effects on bactericidal an immunological activity of blood and skin mucus, and on blood chemistry of Gilthead Seabream ( Sparus aurata ). J. Agr. Aquac., 2(1). Retrieved from https://escientificpublishers.com/assets/data1/images/JAA-02-0018.pdf

Ángeles Esteban, M. (2012). An overview of the immunological defenses in fish skin. ISRN immunol., 2012: 1 - 29. https://doi.org/10.5402/2012/853470

Braun, R.; Arnesen, J. A.; Rinne, A.; and Hjelmeland, K. (1990). Immunohistological localization of trypsin in mucus-secreting cell layers of Atlantic salmon, Salmo salar L. J. Fish Dis., 13(3): 233 - 238. https://doi.org/10.1111/j.1365-2761.1990.tb00778.x

Brokken, L. J. S.; Verbost, P. M.; Atsma, W.; and Wendelaar Bonga, S. E. (1998). Isolation, partial characterization and localization of integumental peroxidase, a stress-related enzyme in the skin of a teleostean fish (Cyprinus carpio L.). Fish Physiol. Biochem., 18(4): 331-342. https://doi.org/10.1023/A:1007707520177

Bulloch, P.; Schur, S.; Muthumuni, D.; Xia, Z.; Johnson, W.; Chu, M.; Palace, V.; Su, G.; Letcher, R.; Tomy, G. T. (2020). F2-isoprostanes in fish mucus: A new, non-invasive method for analyzing a biomarker of oxidative stress. Chemosphere, 239: 124797. https://doi.org/10.1016/j.chemosphere.2019.124797

Cabillon, N. A. R., \& Lazado, C. C. (2019). Mucosal barrier functions of fish under changing environmental conditions. Fishes, 4(1): 1-10. https://doi.org/10.3390/fishes4010002

Charlie-Silva, I.; de Melo, N. F. S.; Gomes, J. M. M.; Fraceto, L. F.; Junior, J. D. C., Conceição, K.; de Andrade Belo, M.A.; Luz, R. K. (2019). Novel nanostructure 
obtained from pacamã, Lophiosilurus alexandri, skin mucus presents potential as a bioactive carrier in fish. Aquaculture, 512(July): 734294. https://doi.org/10.1016/ j.aquaculture.2019.734294

Chaturvedi, P.; Bhat, R. A. H.; and Pande, A. (2020). Antimicrobial peptides of fish: innocuous alternatives to antibiotics. Rev. Aquac., 12(1): 85 - 106. https://doi.org/10.1111/raq.12306

Cipolari, O. C.; de Oliveira Neto, X. A.; and Conceição, K. (2020). Fish bioactive peptides: A systematic review focused on sting and skin. Aquaculture, 515: 734598. https://doi.org/10.1016/j.aquaculture.2019.734598

Cone, R. A. (2009). Barrier properties of mucus. Adv. Drug Deliv. Rev., 61(2): 75-85. https://doi.org/10.1016/j.addr.2008.09.008

Cordero, H.; Cuesta, A.; Meseguer, J.; and Esteban, M. Á. (2016). Changes in the levels of humoral immune activities after storage of gilthead seabream (Sparus aurata) skin mucus. Fish Shellfish Immun., 58: 500 - 507. https://doi.org/10.1016/j.fsi.2016.09.059

Dang, M.; Pittman, K.; Bach, L.; Sonne, C.; Hansson, S. V.; Søndergaard, J.; Stride, M.; Nowak, B. (2019). Mucous cell responses to contaminants and parasites in shorthorn sculpins (Myoxocephalus scorpius) from a former lead-zinc mine in West Greenland. Sci. Total Environ., 678: 207 - 216. https://doi.org/10.1016/j.scitotenv.2019.04.412

Dang, M.; Pittman, K.; Sonne, C.; Hansson, S.; Bach, L.; Søndergaard, J.; Stride, M.; Nowak, B. (2020). Histological mucous cell quantification and mucosal mapping reveal different aspects of mucous cell responses in gills and skin of shorthorn sculpins (Myoxocephalus scorpius). Fish Shellfish Immun., 100: 334 - 344. https://doi.org /10. 1016/j.fsi.2020.03.020

Dash, S.; Das, S. K.; Samal, J.; and Thatoi, H. N. (2018). Epidermal mucus, a major determinant in fish health: A review. Iran J. Vet Res., 19(2): 72 - 81. https://doi.org/ 10.22099/ijvr.2018.4849

Dzul-Caamal, R.; Olivares-Rubio, H. F.; Salazar-Coria, L.; Rocha-Gómez, M. A.; and Vega-López, A. (2016). Multivariate analysis of biochemical responses using noninvasive methods to evaluate the health status of the endangered blackfin goodeid (Girardinichthys viviparus). Ecol. Indic., 60: 1118 - 1129. https://doi.org/10.1016/ j.ecolind.2015.09.017

Dzul-Caamal, R.; Salazar-Coria, L.; Olivares-Rubio, H. F.; Rocha-Gómez, M. A.; GirónPérez, M. I.; and Vega-López, A. (2016). Oxidative stress response in the skin mucus layer of Goodea gracilis (Hubbs and Turner, 1939) exposed to crude oil: A noninvasive approach. Comp. Biochem. Phys. Part A Mol. Integr. Physiol., 200: 9 - 20. https://doi.org/10.1016/j.cbpa.2016.05.008

Easy, R. H.; and Ross, N. W. (2010). Changes in Atlantic salmon Salmo salar mucus components following short- and long-term handling stress. J. Fish Biol., 77(7): 1616 1631. https://doi.org/10.1111/j.1095-8649.2010.02796.x

Easy, R. H.; Trippel, E. A.; Burt, M. D. B.; and Cone, D. K. (2012). Identification of transferrin in Atlantic cod Gadus morhua epidermal mucus. J. Fish Biol., 81(6): 2059 2063. https://doi.org/10.1111/j.1095-8649.2012.03452.x 
Eddy, F. B., and Fraser, J. E. (1982). Sialic acid and mucus production in rainbow trout (Salmo gairdneri Richardson) in response to zinc and seawater. Comp. Biochem. Phys. Part C Mol. Integr. Physiol., 73(2): 357-359. https://doi.org/10.1016/0306-4492(82)90135-6

Ekman, D. R.; Skelton, D. M.; Davis, J. M.; Villeneuve, D. L.; Cavallin, J. E.; Schroeder, A.; Jensen, K.M.; Ankley, G.T.; Collette, T. W. (2015). Metabolite profiling of fish skin mucus: A novel approach for minimally-invasive environmental exposure monitoring and surveillance. Environ. Sci. Technol., 49(5): 3091 - 3100. https://doi.org/10.1021/es505054f

Fæste, C. K.; Tartor, H.; Moen, A.; Kristoffersen, A. B.; Dhanasiri, A. K. S.; Anonsen, J. H.; Furmanek, T.; Grove, S. (2020). Proteomic profiling of salmon skin mucus for the comparison of sampling methods. J. Chromatogr. B, 1138: 121965. https://doi.org/10.1016/j.jchromb.2019.121965

Fagan, M. S.; O'Byrne-Ring, N.; Ryan, R.; Cotter, D.; Whelan, K., and Evilly, U. Mac. (2003). A biochemical study of mucus lysozyme, proteins and plasma thyroxine of Atlantic salmon (Salmo salar) during smoltification. Aquaculture, 222(1 - 4): 287 - 300. https://doi.org/10.1016/S0044-8486(03)00128-5

Fakih, T. M., and Dewi, M. L. (2020). Molecular interaction of yellow catfish (Pelteobagrus fulvidraco) antimicrobial peptide against Penicillin-Binding Protein 3 (PBP3) in Escherichia coli by in silico. Bioeduscience, 04(01): 48 - 55. Retrieved from https://journal.uhamka.ac.id/index.php/bioeduscience/article/view/4951/1865

Fan, C.; Wang, J.; Zhang, X., and Song, J. (2015). Functional C1q is present in the skin mucus of Siberian sturgeon (Acipenser baerii). Integr. Zoo., 10(1): 102 - 110. https://doi.org/10.1111/1749-4877.12100

Fekih-Zaghbib, S.; Fildier, A.; Barrek, S.; \& Bouhaouala-Zahar, B. (2013). A complementary LC-ESI-MS and MALDI-TOF approach for screening antibacterial proteomic signature of farmed European Sea bass mucus. Fish Shellfish Immun., 35(2): 207 - 212. https://doi.org/10.1016/j.fsi.2013.04.017

Fernández-Alacid, L.; Sanahuja, I.; Ordóñez-Grande, B.; Sánchez-Nuño, S.; Herrera, M.; and Ibarz, A. (2019). Skin mucus metabolites and cortisol in meagre fed acute stressattenuating diets: Correlations between plasma and mucus. Aquaculture, 499: 185 - 194. https://doi.org/10.1016/j.aquaculture.2018.09.039

Fernández-Alacid, L.; Sanahuja, I.; Ordóñez-Grande, B.; Sánchez-Nuño, S.; Viscor, G.; Gisbert, E.; Herrera, M.; Ibarz, A. (2018). Skin mucus metabolites in response to physiological challenges: A valuable non-invasive method to study teleost marine species. Sci. Total Environ., 644: 1323 - 1335. https://doi.org/10.1016/j. scitotenv. 2018.07.083

Fernández-Montero, A.; Torrecillas, S.; Tort, L.; Ginés, R.; Acosta, F.; Izquierdo, M. S.; and Montero, D. (2020). Stress response and skin mucus production of greater amberjack (Seriola dumerili) under different rearing conditions. Aquaculture, 520(January): 735005. https://doi.org/10.1016/j.aquaculture.2020.735005

Ghalambor, M.; Eslamifar, Z.; and Khoshnood, Z. (2020). Biochemical characterization of lysozyme extracted from Common Carp, Cyprinus carpio. Ecopersia, 8(2): 125 -131.

Gobi, N.; Vaseeharan, B.; Chen, J. C.; Rekha, R.; Vijayakumar, S.; Anjugam, M., and Iswarya, A. (2018). Dietary supplementation of probiotic Bacillus licheniformis Dahb1 
improves growth performance, mucus and serum immune parameters, antioxidant enzyme activity as well as resistance against Aeromonas hydrophila in tilapia Oreochromis mossambicus. Fish Shellfish Immun., 74(2018): 501 - 508. https://doi.org/10.1016/j.fsi.2017.12.066

Gokhale, A. S., and Satyanarayanajois, S. (2014). Peptides and peptidomimetics as unomodulators. Immunotherapy, 6(6): 755 - 774. immhttps://doi.org/10.2217/imt.14.37

Guardiola, F. A.; Cuartero, M.; del Mar Collado-González, M.; Díaz Baños, F. G.; Cuesta, A.; Moriñigo, M. Á.; and Esteban, M. Á. (2017). Terminal carbohydrates abundance, immune related enzymes, bactericidal activity and physico-chemical parameters of the Senegalese sole (Solea senegalensis, Kaup) skin mucus. Fish Shellfish Immun., 60: 483 - 491. https://doi.org/10.1016/j.fsi.2016.11.025

Guardiola, F. A.; Cuesta, A.; Abellán, E.; Meseguer, J.; and Esteban, M. A. (2014). Comparative analysis of the humoral immunity of skin mucus from several marine teleost fish. Fish Shellfish Immun., 40(1): 24 - 31. https://doi.org/10.1016/ j.fsi. 2014.06.018

Guardiola, F. A.; Cuesta, A.; Arizcun, M.; Meseguer, J.; and Esteban, M. A. (2014). Comparative skin mucus and serum humoral defence mechanisms in the teleost gilthead seabream (Sparus aurata). Fish Shellfish Immun., 36(2): 545 - 551. https://doi.org/10.1016/j.fsi.2014.01.001

Guardiola, F. A.; Cuesta, A.; and Esteban, M. Á. (2016). Using skin mucus to evaluate stress in gilthead seabream (Sparus aurata L.). Fish Shellfish Immun., 59: 323 - 330. https://doi.org/10.1016/j.fsi.2016.11.005

Guardiola, F. A.; Dioguardi, M.; Parisi, M. G.; Trapani, M. R.; Meseguer, J.; Cuesta, A.; Cammarata, M.; Esteban, M. A. (2015). Evaluation of waterborne exposure to heavy metals in innate immune defences present on skin mucus of gilthead seabream (Sparus aurata). Fish Shellfish Immun., 45(1): 112 - 123. https://doi.org/10.1016/ j.fsi. 2015 02.010

Gustafsson, J. K., Navabi, N., Rodriguez-Piñeiro, A. M., Alomran, A. H. A., Premaratne, P.; Fernandez, H. R.; Banerjee, D.; Sjovall, H.; Hansson, G.C.; Lindén, S. K. (2013). Dynamic changes in mucus thickness and ion secretion during citrobacter rodentium infection and clearance. PLoS ONE, 8(12). https://doi.org/10.1371/ journal.pone. 0084430

Iger, Y.; Jenner, H. A.; and Bonga, S. E. W. (1994). Cellular responses in the skin of the trout (Oncorhynchus mykiss) exposed to temperature elevation. J. Fish Biol., 44(6): 921 935. https://doi.org/10.1111/j.1095-8649.1994.tb01265.x

Kumar, P.; Rajeshwaran, T.; Priya, P.; Kailasam, M.; Biswas, G.; Ghoshal, T. K.; Vijayan, K.K.; Arasu, A. R. T. (2019). Comparative immunological and biochemical properties of the epidermal mucus from three brackish water fishes. Proc. Natl. Acad. Sci. India Sect. B Biol. Sci., 89(1): 95 - 103. https://doi.org/10.1007/s40011-017-0923-3

Lai, S. K.; Wang, Y. Y.; Wirtz, D.; and Hanes, J. (2009). Micro- and macrorheology of mucus. Adv. Drug Deliv. Rev., 61(2): 86 - 100. https://doi.org/10.1016/j.addr.2008.09.012

Lallès, J. P. (2019). Biology, environmental and nutritional modulation of skin mucus alkaline phosphatase in fish: A review. Fish Shellfish Immun., 89: 179 - 186. https://doi.org/10.1016/j.fsi.2019.03.053 
Lei, J.; Sun, L. C.; Huang, S.; Zhu, C.; Li, P.; He, J.; Coy, D.H.; He, Q. Y. (2019). The antimicrobial peptides and their potential clinical applications. Am. J. Transl. Res., 11(7): 3919 - 3931.

Liu, H. han; Sun, Q.; Jiang, Y. ting; Fan, M. hua; Wang, J. xin; and Liao, Z. (2019). Indepth proteomic analysis of Boleophthalmus pectinirostris skin mucus. J. Proteomics, 200: 74 - 89. https://doi.org/10.1016/j.jprot.2019.03.013

Liu, L.; Li, C.; Su, B.; Beck, B. H.; and Peatman, E. (2013). Short-term feed deprivation alters immune status of surface mucosa in Channel Catfish (Ictalurus punctatus). PLoS ONE, 8(9): 1 - 10. https://doi.org/10.1371/journal.pone.0074581

Magnadóttir, B.; Kraev, I.; Guðmundsdóttir, S.; Dodds, A. W.; and Lange, S. (2019). Extracellular vesicles from cod (Gadus morhua L.) mucus contain innate immune factors and deiminated protein cargo. Dev. Comp. Immunol., 99(April): 103397. https://doi.org/10.1016/j.dci.2019.103397

Mahadevan, G.; Mohan, K.; Vinoth, J.; and Ravi, V. (2019). Biotic potential of mucus extracts of giant mudskipper Periophthalmodon schlosseri (Pallas, 1770) from Pichavaram, southeast coast of India. J. Basic Appl. Zool., 80(1). https://doi.org/10.1186/s41936-019-0084-4

Meucci, V., and Arukwe, A. (2005). Detection of vitellogenin and zona radiata protein expressions in surface mucus of immature juvenile Atlantic salmon (Salmo salar) exposed to waterborne nonylphenol. Aquat. Toxicol., 73(1): 1 - 10. https://doi.org/10.1016/j.aquatox.2005.03.021

Montenegro, D.; Astudillo-García, C.; Hickey, T.; and Lear, G. (2020). A non-invasive method to monitor marine pollution from bacterial DNA present in fish skin mucus. Environ. Pollut., 263: 114438. https://doi.org/10.1016/j.envpol.2020.114438

Nigam, A. K; Kumari, U.; Mittal, S.; and Mittal, A. (2014). Characterization of carboxylesterase in skin mucus of Cirrhinus mrigala and its assessment as biomarker of organophosphate exposure. Fish Physiol. Biochem., 40(3): 635 - 644. https://doi.org/10.1007/s10695-013-9872-9

Nigam; Ashwini Kumar; Srivastava, N.; Rai, A. K.; Kumari, U.; Mittal, A. K., and Mittal, S. (2014). The first evidence of cholinesterases in skin mucus of Carps and its applicability as biomarker of organophosphate exposure. Environ. Toxicol., 29(7): 788 796. https://doi.org/10.1002/tox

Oliveira, M.; Tvarijonaviciute, A.; Trindade, T.; Soares, A. M. V. M.; Tort, L., and Teles, M. (2018). Can non-invasive methods be used to assess effects of nanoparticles in fish?. Ecol. Indic., 95(June): 1118 - 1127. https://doi.org/10.1016/j.ecolind.2017.06.023

Omidi, F.; Jafaryan, H.; Patimar, R.; Harsij, M.; and Paknejad, H. (2020). Biochemical biomarkers of skin mucus in Neogobius melanostomus for assessing lead pollution in the Gulf of Gorgan (Iran). Toxicol. Rep., 7(December 2019): 109 - 117. https://doi.org/10.1016/j.toxrep.2019.12.003

Ouyang, M. Y.; Wen, B.; Ma, H. C.; Chen, C.; Gao, J. Z.; Zhang, Y.; and Chen, Z. Z. (2020). Minimally invasive evaluation of the anaesthetic efficacy of MS-222 for ornamental discus fish using skin mucus biomarkers. Aquac. Res., (January): 1 - 10. https://doi.org/10.1111/are.14631 
Palaksha, K. J.; Shin, G. W.; Kim, Y. R.; and Jung, T. S. (2008). Evaluation of non-specific immune components from the skin mucus of olive flounder (Paralichthys olivaceus). Fish Shellfish Immun., 24(4): 479 - 488. https://doi.org/10.1016/j.fsi.2008.01.005

Patel, D. M., and Brinchmann, M. F. (2017). Skin mucus proteins of lumpsucker (Cyclopterus lumpus). Biochem. Biophys. Rep., 9(August 2016): $217 \quad$ - 225. https://doi.org/10.1016/j.bbrep.2016.12.016

Patel, M.; Ashraf, M. S.; Siddiqui, A. J.; Ashraf, S. A.; Sachidanandan, M.; Snoussi, M.; Adnan, M.; and Hadi, S. (2020). Profiling and role of bioactive molecules from puntius sophore (Freshwater/brackish fish) skin mucus with its potent antibacterial, antiadhesion, and antibiofilm activities. Biomolecules, 10(6): 1 - 27. https://doi.org/10.3390/biom10060920

Pérez-Sánchez, J.; Terova, G.; Simó-Mirabet, P.; Rimoldi, S.; Folkedal, O.; Calduch-Giner, J. A.; Olsen, S.E.; Sitjà-Bobadilla, A. (2017). Skin mucus of gilthead sea bream (Sparus aurata L.) protein mapping and regulation in chronically stressed fish. Front. Physiol., 8(February): 1 - 18. https://doi.org/10.3389/fphys.2017.00034

Pietrzak, E.; Mazurkiewicz, J.; and Slawinska, A. (2020). Innate immune responses of skin mucosa in common carp (Cyprinus carpio) fed a diet supplemented with galactooligosaccharides. Animals, 10(3). https://doi.org/10.3390/ani10030438

Rajan, B., Lokesh, J., Kiron, V., and Brinchmann, M. F. (2013). Differentially expressed proteins in the skin mucus of Atlantic cod (Gadus morhua) upon natural infection with Vibrio anguillarum. BMC Vet. Res., 9. https://doi.org/10.1186/1746-6148-9-103

Reverter, M.; Tapissier-Bontemps, N.; Lecchini, D.; Banaigs, B.; and Sasal, P. (2018). Biological and ecological roles of external fish mucus: A review. Fishes, 3(4): 41. https://doi.org/10.3390/fishes3040041

Ross, N. W.; Firth, K. J.; Wang, A.; Burka, J. F.; and Johnson, S. C. (2000). Changes in hydrolytic enzyme activities of native Atlantic salmon Salmo salar skin mucus due to infection with the salmon louse Lepeophtheirus salmonis and cortisol implantation. Dis. Aquat. Organ., 41(1): 43 - 51. https://doi.org/10.3354/dao041043

Rusli, N., and Yeniati, N. (2019). Formulation of Catfish slime gel formation (Clarias gariepinus L) as a wound healer with a variety of carbopol base 934. Medical Sains, 3(2): 131 - 138. Retrieved from http://ojs.stfmuhammadiyahcirebon. ac.id/index. php/iojs/article/view/57/71

Safaruddin; Safitri, N. A.; Yuliana, B.; and Firman, I. (2019). Formulation of mucus fish gel (Channa striata) and effectiveness test as a burn medicine for rabbits (Oryctolagus cuniculus). UIT 2019 National Seminar on Science, Technology, and Social Humanities. Retrieved from the Research and Community Service Institute. Makassar Eastern Indonesia University

Salinas, I. (2015). The mucosal immune system of teleost fish. Biology, 4(3): 525 - 539. https://doi.org/10.3390/biology4030525

Sanahuja, I., and Ibarz, A. (2015). Skin mucus proteome of gilthead sea bream: A non-invasive method to screen for welfare indicators. Fish Shellfish Immun., 46(2): 426 - 435. https://doi.org/10.1016/j.fsi.2015.05.056

Sheikhzadeh, N.; Karimi Pashaki, A.; Nofouzi, K.; Heidarieh, M.; and Tayefi-Nasrabadi, H. (2012). Effects of dietary ergosan on cutaneous mucosal immune response in 
rainbow trout (Oncorhynchus mykiss). Fish Shellfish Immun., 32(3): 407 - 410. https://doi.org/10.1016/j.fsi.2011.11.028

Shen, Y.; Zhang, J.; Xu, X.; Fu, J.; and Li, J. (2012). Expression of complement component $\mathrm{C} 7$ and involvement in innate immune responses to bacteria in grass carp. Fish Shellfish Immun., 33(2): 448 - 454. https://doi.org/10.1016/j.fsi.2012.05.016

Shephard, K. L. (1993). Mucus on the epidermis of fish and its influence on drug delivery. Adv. Drug Deliv. Rev., 11(3): 403 - 417. https://doi.org/10.1016/0169-409X(93)90018-Y

Srichaiyo, N.; Tongsiri, S.; Hoseinifar, S. H.; Dawood, M. A. O.; Jaturasitha, S.; Esteban, M. Á.; Ringo, E.; Van Doan, H. (2020). The effects gotu kola (Centella asiatica) powder on growth performance, skin mucus, and serum immunity of Nile tilapia (Oreochromis niloticus) fingerlings. Aquacult. Rep., 16(August 2019): 100239. https://doi.org/10.1016/j.aqrep.2019.100239

Srivastava, A., Nigam, A. K., Mittal, S., and Mittal, A. K. (2018). Role of aloin in the modulation of certain immune parameters in skin mucus of an Indian major carp, Labeo rohita. Fish Shellfish Immun., 73: 252-261. https://doi.org/10.1016/j.fsi.2017.12.014

Subramanian, S.; MacKinnon, S. L.; and Ross, N. W. (2007). A comparative study on innate immune parameters in the epidermal mucus of various fish species. Comp. Biochem. Physiol. B Biochem. Mol. Biol., 148(3): 256 - 263. https://doi.org/10.1016/ j.cbpb.2007.06.003

Subramanian, S.; Ross, N. W.; and MacKinnon, S. L. (2008). Comparison of antimicrobial activity in the epidermal mucus extracts of fish. Comp. Biochem. Physiol. B Biochem. Mol. Biol., 150(1): 85 - 92. https://doi.org/10.1016/j.cbpb.2008.01.011

Sudaryatma, P. E.; Lestari, A. T.; Luh, S. N.; Widiarti, K. S.; Hidayah, N., and Srinoto, D. (2012). Immunocytochemistry streptavidin biotin: Early detection of viral nervous necrosis virus in the mucous of the ikan Kerapu Macan (Epinephelus fuscoguttatus). JSV., 30(1), 99-109. https://doi.org/10.22146/jsv.2487

Sugiyama, N.; Araki, M.; Ishida, M.; Nagashima, Y.; and Shiomi, K. (2005). Further isolation and characterization of grammistins from the skin secretion of the soapfish Grammistes sexlineatus. Toxicon, 45(5): 595 - 601. https://doi.org/10.1016/ j.toxicon. 2004.12.021

Sunyer, J. O. (2013). Fishing for mammalian paradigms in the teleost immune system. Nat. Immunol., 14(4): 320 - 326. https://doi.org/10.1038/ni.2549

Tapia-Paniagua, S. T.; Ceballos-Francisco, D.; Balebona, M. C.; Esteban, M. Á., and Moriñigo, M. Á. (2018). Mucus glycosylation, immunity and bacterial microbiota associated to the skin of experimentally ulcered gilthead seabream (Sparus aurata). Fish Shellfish Immun., 75(January): 381 - 390. https://doi.org/10.1016/j.fsi.2018.02.006

Tarnawska, M.; Augustyniak, M.; Laszczyca, P.; Migula, P.; Irnazarow, I.; Krzyżowski, M.; and Babczyńska, A. (2019). Immune response of juvenile common carp (Cyprinus carpio L.) exposed to a mixture of sewage chemicals. Fish Shellfish Immun., 88(February): 17 - 27. https://doi.org/10.1016/j.fsi.2019.02.049

Terova, G.; Cattaneo, A. G.; Preziosa, E.; Bernardini, G., and Saroglia, M. (2011). Impact of acute stress on antimicrobial polypeptides mRNA copy number in several tissues of marine sea bass (Dicentrarchus labrax). BMC Immunol., 12. https://doi.org/ $10.1186 / 1471-2172-12-69$ 
Timalata, K.; Marimuthu, K.; Vengkades Rao, R.; Xavier, R.; Rahman, M. A.; Sreeramanan, S.; Al-Dhabi, N.A.; Arockiaraj, J. (2015). Elucidation of innate immune components in the epidermal mucus of different freshwater fish species. Acta Ichthyol. Piscat., 45(3): 221 - 230. https://doi.org/10.3750/AIP2015.45.3.01

Valero, Y.; Cortés, J., and Mercado, L. (2019). NK-lysin from skin-secreted mucus of Atlantic salmon and its potential role in bacteriostatic activity. Fish Shellfish Immun., 87(January): 410 - 413. https://doi.org/10.1016/j.fsi.2019.01.034

van der Marel M.; Caspari, N.; Neuhaus, H.; Meyer, W.; Enss, M. L., and Steinhagen, D. (2010). Changes in skin mucus of common carp, Cyprinus carpio L., after exposure to water with a high bacterial load. J. Fish Dis., 33(5): 431 - 439. https://doi.org/10.1111/j.1365-2761.2010.01140.x

Van Doan, H.; Hoseinifar, S. H.; Sringarm, K.; Jaturasitha, S.; Yuangsoi, B.; Dawood, M. A. O.; Esteban, M.A.; Ringo, E.; Faggio, C. (2019). Effects of assam tea extract on growth, skin mucus, serum immunity and disease resistance of Nile tilapia (Oreochromis niloticus) against Streptococcus agalactiae. Fish Shellfish Immunol., 93(July): 428 - 435. https://doi.org/10.1016/j.fsi.2019.07.077

Wang, H.; Tang, W.; Zhang, R., and Ding, S. (2019). Analysis of enzyme activity, antibacterial activity, antiparasitic activity and physico-chemical stability of skin mucus derived from Amphiprion clarkii. Fish Shellfish Immunol., 86: 653 - 661. https://doi.org/10.1016/j.fsi.2018.11.066

Xia, H.; Liu, W.; Wu, K.; Wang, W., and Zhang, X. (2016). sIgZ exhibited maternal transmission in embryonic development and played a prominent role in mucosal immune response of Megalabrama amblycephala. Fish Shellfish Immunol., 54: 107 117. https://doi.org/10.1016/j.fsi.2016.03.165

Xiong, Y.; Dan, C.; Ren, F.; Su, Z. H.; Zhang, Y., and Mei, J. (2020). Proteomic profiling of yellow catfish (Pelteobagrus fulvidraco) skin mucus identifies differentially-expressed proteins in response to Edwardsiella ictaluri infection. Fish Shellfish Immunol., (Vol. 100). https://doi.org/10.1016/j.fsi.2020.02.059

Xu, Z.; Parra, D.; Gómez, D.; Salinas, I.; Zhang, Y. A.; Von Gersdorff Jørgensen, L.; Heinecke, R.D.; Buchmann, K.; LaPatra, S.; Oriol Sunyer, J. (2013). Teleost skin, an ancient mucosal surface that elicits gut-like immune responses. PNAS USA., 110(32): 13097 - 13102. https://doi.org/10.1073/pnas.1304319110 